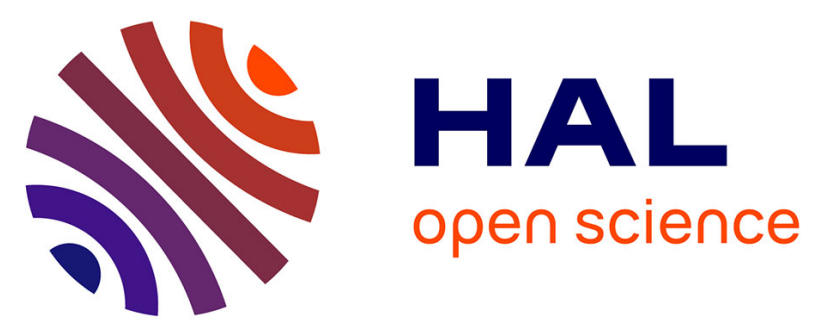

\title{
Une fosse de l'Âge du Bronze final dans la grotte de la Baume des Anges à Donzère (Drôme)
}

Joël Vital, Louis Chaix, Philippe Marinval, Stéphanie Thiébault

\section{To cite this version:}

Joël Vital, Louis Chaix, Philippe Marinval, Stéphanie Thiébault. Une fosse de l'Âge du Bronze final dans la grotte de la Baume des Anges à Donzère (Drôme): Annexe 1: La faune de la baune des anges (Drôme, France) (Bronze final). Annexe 2: Analyse paléocarpologique: baume des anges. Donzère (Drôme), Age du bronze final. Annexe 3: Analyse des chabons de bois de la grotte de la Baume des anges.. Bulletin de la Société préhistorique française, 1986, 83 (1), pp.17-32 + (3 annexes). halshs-03344216

\section{HAL Id: halshs-03344216 https://shs.hal.science/halshs-03344216}

Submitted on 14 Sep 2021

HAL is a multi-disciplinary open access archive for the deposit and dissemination of scientific research documents, whether they are published or not. The documents may come from teaching and research institutions in France or abroad, or from public or private research centers.
L'archive ouverte pluridisciplinaire HAL, est destinée au dépôt et à la diffusion de documents scientifiques de niveau recherche, publiés ou non, émanant des établissements d'enseignement et de recherche français ou étrangers, des laboratoires publics ou privés. 


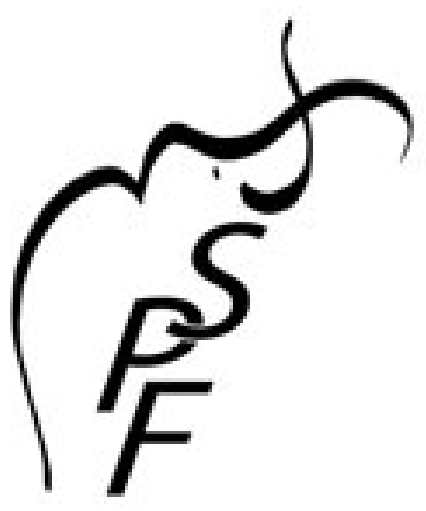

Une fosse de l'Age du Bronze final dans la grotte de la Baume des Anges à Donzère (Drôme) Author(s): Joël Vital, Louis Chaix, Philippe Marinval and Stéphanie Thiebault

Reviewed work(s):

Source: Bulletin de la Société préhistorique française, T. 83, No. 1 (1986), pp. 17-32

Published by: Société Préhistorique Française

Stable URL: http://www.jstor.org/stable/27918907

Accessed: $27 / 08 / 2012$ 10:39

Your use of the JSTOR archive indicates your acceptance of the Terms \& Conditions of Use, available at http://www.jstor.org/page/info/about/policies/terms.jsp

JSTOR is a not-for-profit service that helps scholars, researchers, and students discover, use, and build upon a wide range of content in a trusted digital archive. We use information technology and tools to increase productivity and facilitate new forms of scholarship. For more information about JSTOR, please contact support@ jstor.org. 


\title{
Une fosse de l'Age du Bronze final dans la grotte de la Baume des Anges à Donzère (Drôme)
}

\author{
par Joël Vital
}

\begin{abstract}
Résumé : L'analyse des matériaux contenus dans une fosse d'extraction d'argile de la grotte de la Baume des Anges à Donzère permet de montrer que cette structure a été utilisée comme dépotoir à deux reprises et comme aire d'implantation d'un foyer. L'étude des objets recueillis est prétexte à évoquer certains points de la chronologie du Bronze final 2 et $3 b$ de la région.
\end{abstract}

Summary : The analysis of the materials enclosed in a clay extraction pit of the cave Baume des Anges at Donzère (Drôme) proves this structure has been used as a refuse dump on two occasions and as a place for the installation of a hearth. The study of the collected objects allows us to see certains points of the chronology of the local 2 and $3 b$ late Bronze age.

Riassunto : Lo studio dei materiali contenuti in una fossa d'estrazione d'argilla nella caverna della Baume des Anges di Donzère (Drôme) dimostra che questa struttura fosse utilizzata come scarico a due represe e come luogo d'impiantato di un focolaio. Lo studio dei ogetti raccogliati e pretesta ad evocare certi punti della cronologia del Bronzo finale 2 e 3 b locale.

\section{1. - SITUATION}

Située à mi-distance de Valence et d'Avignon (fig. 1), la grotte de la Baume des Anges s'ouvre dans une falaise subverticale dont la base est masquée sous un important éboulis (fig. 2) et qui constituent la rive gauche du Rhône au lieudit le Défilé de Donzère. Le modelé actuel de cette microrégion est récent, puisque la percée par le fleuve des calcaires urgoniens (Crétacé inférieur) de l'anticlinal de Viviers, d'axe est-ouest, semble due à un creusement épigénique lié aux cycles glaciaires et interglaciaires du Riss et du Würm (Demarcq, 1973, pp. 128 et 143 ; Bornand, Combier, Lorenchet de Montjamont et al., 1979). Il ne faut pas oublier dans cette genèse la part qui revient au faisceau des failles cévenoles (Debelmas, 1974 , pp. 510 et 519) et à la présence d'un important réseau karstique, en grande partie fossile actuellement. Le Défilé de Donzère marque la limite septentrionale du monde méditerranéen et de ses caractéristiques climatiques; il sépare la région dauphinoise du Comtat et de la Provence.

\section{2. - HISTORIQUE DES RECHERCHES}

La Baume des Anges est l'une des cinq grottes occupées durant la Protohistoire dans le Défilé et compte parmi les trois sites qui ont fait l'objet de fouilles récentes. Cette cavité ainsi que les grottes de la Chauve-Souris et des Crapauds (fig. 1) étaient les seules qui recelaient des niveaux archéologiques, plus ou moins bouleversés par les travaux antérieurs, la seconde restant la seule actuellement en cours de fouilles (Vital, 1982 et 1984).

C'est depuis plus d'un siècle que les visiteurs se succèdent dans les grottes donzéroises et leur attention s'est surtout portée sur la plus riche : la Baume des Anges. Nos prédécesseurs immédiats ont avant tout œuvré dans la salle principale, entre 1967 et 1974 (fig. 2), qui leur a livré une stratigraphie où étaient représentés des niveaux du Néolithique final à l'époque gallo-romaine (Lambert et coll., 1976 ; Lambert, 1980). Les phases les mieux attestées parmi l'abondant mobilier archéologique sont le début et la fin du Bronze final (BF 1-2a et BF 3b) (Vital, 1984). 


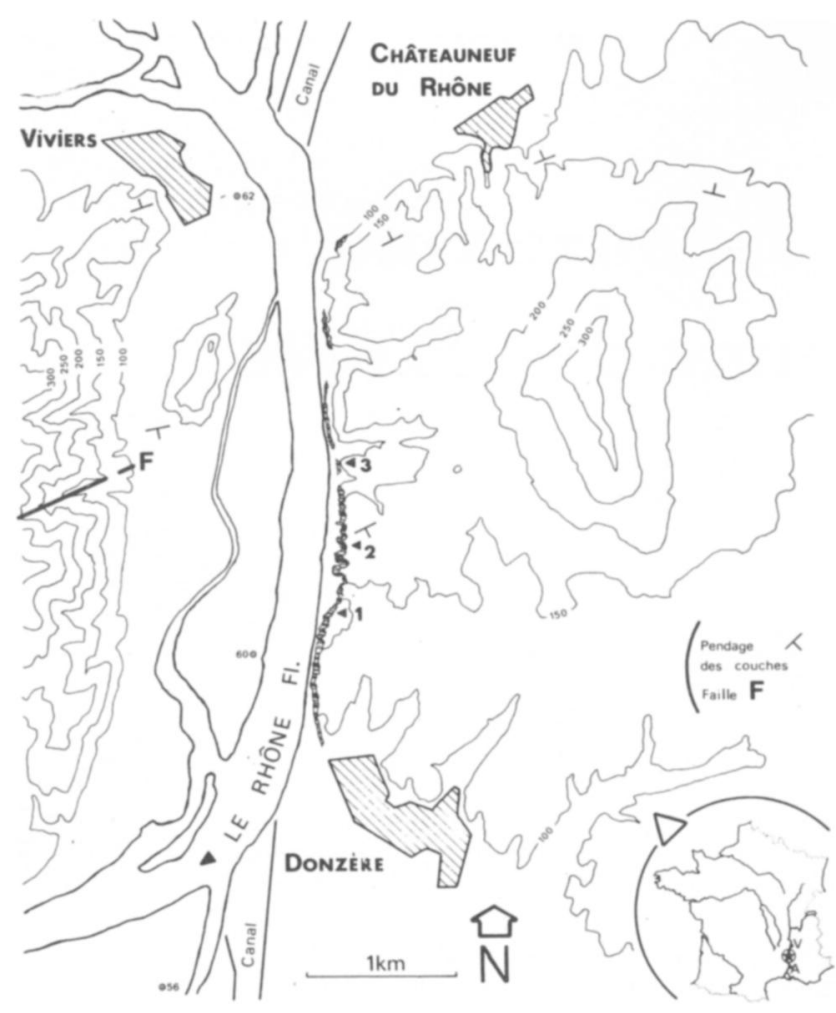

Fig. 1 - Plan de situation du Défilé de Donzère (Drôme) et des principales cavités s'ouvrant en rive gauche du Rhône ; 1 : grotte de la Baume des Anges ; 2 : grotte de la Chauve-Souris ; 3 : grotte des Crapauds.

Plusieurs contraintes, remaniements antérieurs, complexité de la stratigraphie, etc., avaient empêché les fouilleurs d'étudier avec la précision souhaitée les multiples occupations et les vestiges qu'elles livrèrent. Afin de parvenir au reclassement du mobilier exhumé alors, nous avons entrepris en 1982 de retrouver les coupes dégagées par nos devanciers, sans parvenir à notre but, le remplissage ayant subi entre-temps des assauts non coordonnés. Néanmoins l'intervalle compris entre le diverticule, le couloir d'accès au réseau et le sondage $\mathrm{A}$ (fig. 2 et $3 \mathrm{~A}$ ) était susceptible de livrer des couches intactes, selon $M$. Lambert. Les fouilles conduites dans cette zone ont révélé l'existence d'une fosse creusée dans le substratum argilo-sableux et en partie tronquée par l'excavation du sondage $\mathrm{E}$ (1).

\section{3. - LA FOSSE}

Elle était de forme ovale et mesurait $1,50 \mathrm{~m}$ de longueur sur $0,80 \mathrm{~m}$ de largeur environ, si l'on tient

(1) La publication relative aux fouilles anciennes de la grotte de la Baume des Anges est en cours. compte de la partie tronquée anciennement par le sondage $\mathrm{E}$ (fig. $3 \mathrm{~A}$ et 4 ), ses limites étant nettement marquées. La stratigraphie et la répartition des sédiments, piégés sur $30 \mathrm{~cm}$ d'épaisseur, s'établissent comme suit, à partir du substratum composé d'argiles ocre à passées sableuses (fig. 3 B et 4) (2) :

- Couche 6: lentilles cendreuses et amas de limons orangés localisés, en contact avec les argiles sous-jacentes. Rare industrie : 8 tessons (soit $80 \mathrm{~g}$ ) et deux restes osseux (annexe 1).

- Couche 5 : limons argileux bruns hétérogènes, cendreux et peu charbonneux, renfermant des nodules d'argile ocre et des limons orangés compacts. La fraction" fine contient beaucoup de détritique quartzeux et calcaire alors que la fraction grossière est composée d'une majorité de cailloux d'allure gélive (remaniement d'un niveau plus ancien ou gélivation d'époque ?), de très rares cailloux à perforations de lichens et provenant de l'extérieur, et une abondance normale des granules, très rares dans les horizons supérieurs. Les cailloux $(10 \%)$ sont inférieurs à $10 \mathrm{~cm}$. 94 tessons (soit $1840 \mathrm{~g}$ ) du Bronze final 2 sont mêlés sans ordre aux sédiments et à 11 fragments osseux.

- Couche $4 \mathrm{~d}$ : semblable à la précédente mais avec moins de blocs, stérile.

- Couche 4c : limons cendreux et charbonneux gris dans lesquels la part du détritique quartzeux et calcaire est importante. Ils renferment environ $30 \%$ de cailloux et cailloutis de 0,5 à $3 \mathrm{~cm}$, vifs et émoussés. Parmi 129 tessons (soit $2760 \mathrm{~g}$ ) qui peuvent être rapportés au Bronze final 2, une quarantaine proviennent d'un grand récipient fragmenté (fig. 6). Ils reposaient à plat sur une surface cendreuse et sont localisés dans la partie est de la fosse (fig. 4). Ce niveau se sépare bien du précédent et il a livré 10 restes osseux. Trois raccords ont pu être réalisés entre des céramiques des couches $4 c$ et 5 ; les vestiges de 5 récipients différents de forme indéterminée sont également répartis dans ces deux horizons et rassemblent 37 tessons (soit $180 \mathrm{~g}$ ).

- Couche 4b : la fraction fine, détritique, est constituée de limons ocre orangé compacts qui comprennent des nodules cendreux et une abondance de galets de 1 à $3 \mathrm{~cm}$ provenant d'une vieille formation alluviale (soit par le karst, soit par remaniement ou par transport par l'homme), la rareté des éléments à petit diamètre s'accordant peu avec l'hypothèse d'un apport naturel. Cet horizon très localisé est stérile.

- Couche 4a : masse cendreuse très pure et limoneuse, aérée, très charbonneuse et renfermant 3 tessons ; elle occupe une surface égale à celles des

(2) Les sédiments ont été analysés par J.-E. Brochier, Laboratoire de Préhistoire de la Faculté des Sciences Saint-Charles, 13331 Marseille Cedex 3. 
deux niveaux précédents. Quelques céréales ont été recueillies dans les cendres (annexe 2), ainsi que 6 restes osseux.

Couche 4 : limons argileux gris charbonneux comprenant quelques blocs de 3 à $10 \mathrm{~cm}, 76$ tessons (soit $560 \mathrm{~g}$ ) de la phase terminale du Bronze final (BF 3b) et plusieurs fragments d'argile cuite lissés sur une face (restes épars d'une sole de cuisson démantelée), ainsi que 97 fragments d'os.

- Couches 3 à 1 : concrétions calcaires vacuolaires litées (c3 et c1) encadrant un horizon limoneux et cendreux brun foncé très charbonneux (c2). Les
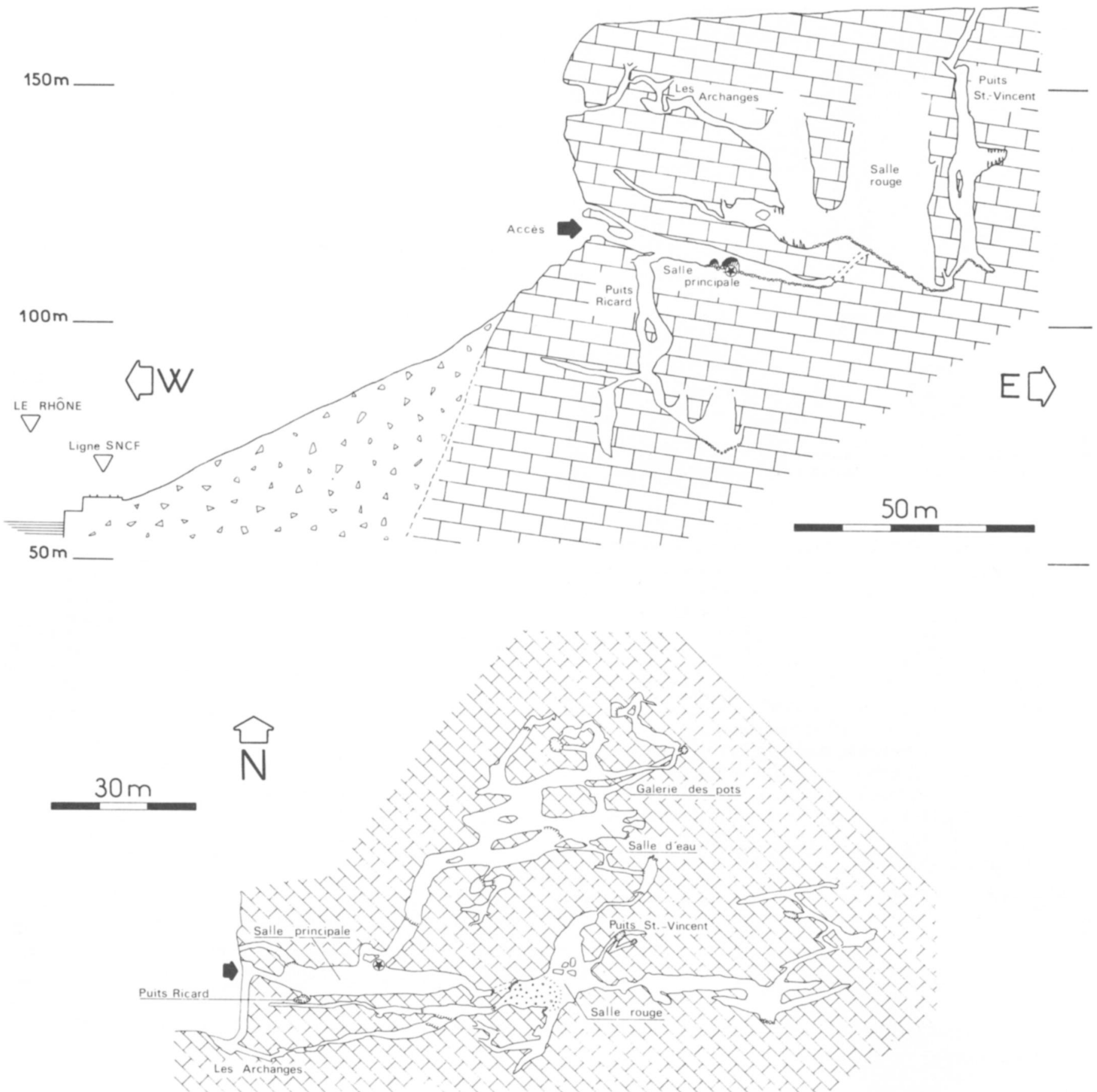

Fig. 2 - Coupe du massif calcaire urgonien constituant la rive gauche, au niveau du réseau de la Baume des Anges, dont le plan montre l'extension générale. L'étoile indique l'emplacement de la zone étudiée. 


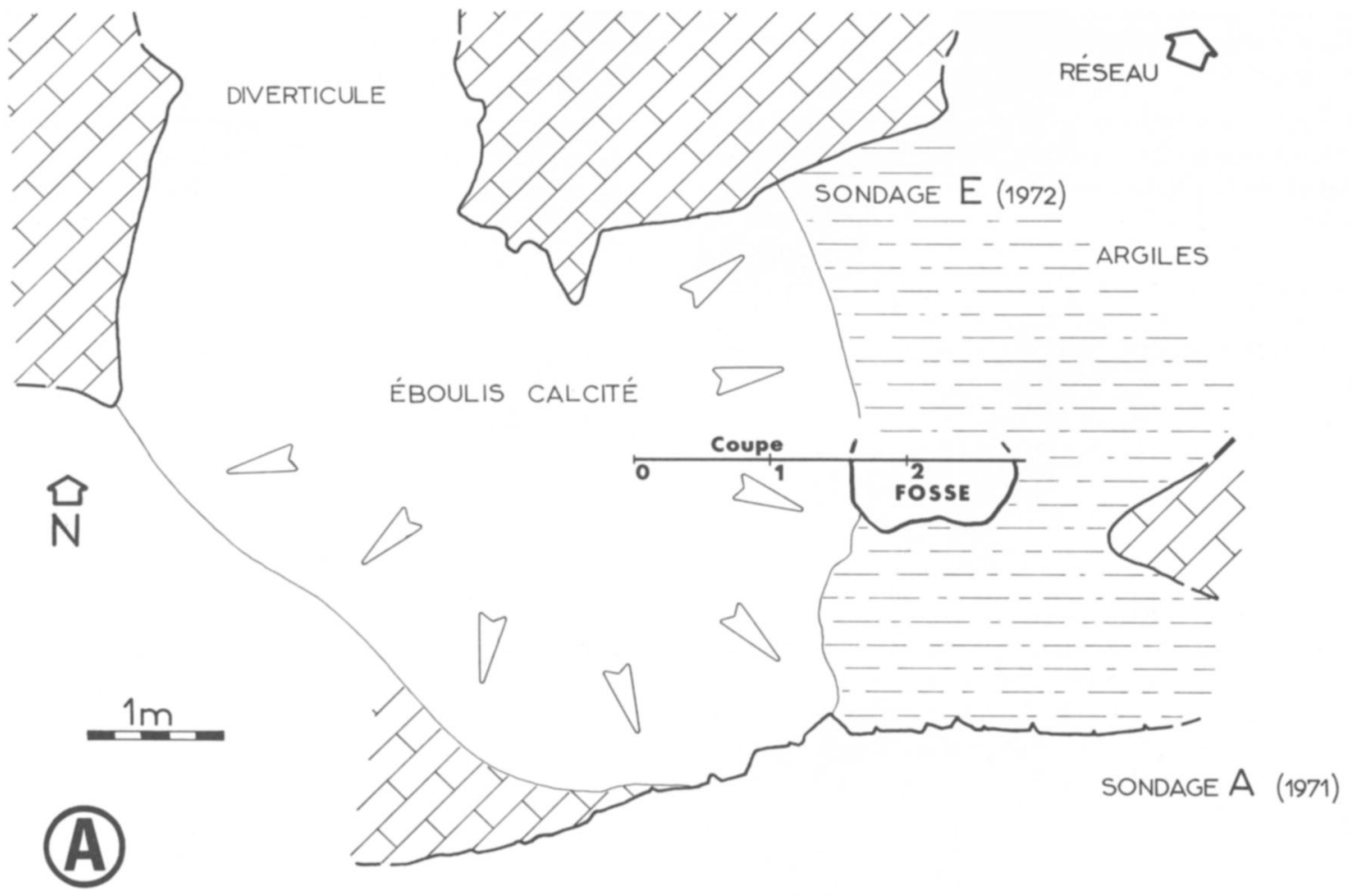

$2 \mid$

(B)

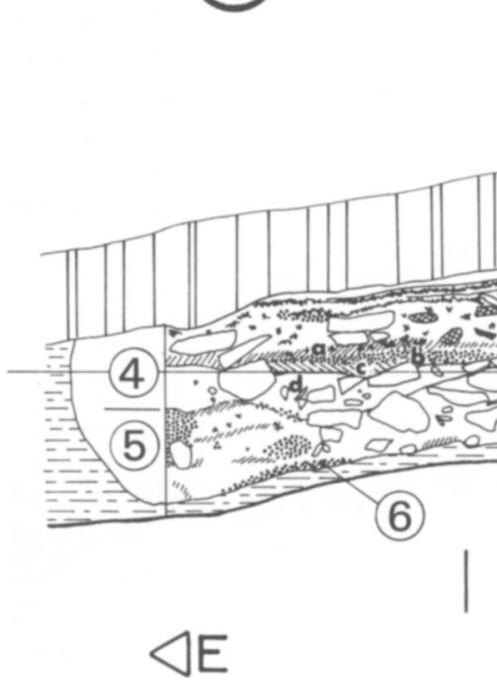

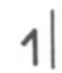

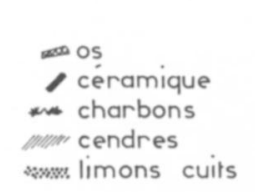
saun limons cuits

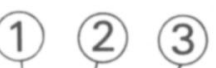


couches 2 et 1 ont livré respectivement 33 et 13 tessons dont plusieurs, que l'on peut rapporter au Bronze moyen et à différents stades du Bronze final, étaient associés.

Dans la totalité de ces formations, sauf en 4a, des traces de crottes d'ovi-capridés ont été révélées par analyse microscopique, mais leur trop faible représentation ne permet pas de conclure à la présence effective d'animaux mais plutôt à une pollution par piétinement, d'autant que l'étude de la grotte des Crapauds, distante de $1000 \mathrm{~m}$, révèle qu'elle a été utilisée principalement en bergerie (Vital, 1984 et à paraître).

\section{1. - Interprétation}

Les observations effectuées sur cette structure et son histoire peuvent être ainsi résumées. Sa morphologie n'évoquant pas une genèse naturelle, il est probable que ce sont les occupants de la grotte qui ont creusé la fosse dans le substratum argileux, en respectant le contour de l'éboulis calcité qui la borde à l'ouest.

L'hétérogénéité des couches $4 \mathrm{~d}$ à 6 qui renferment des vestiges de natures variées, évoque un mode de mise en place rapide. Les parois de la fosse et le mobilier ne portant aucun stigmate de cuisson, il apparaît que les cendres et les charbons résultent non d'une combustion sur place mais plutôt du nettoyage d'une aire d'habitat contiguë, leur dissémination renforçant cette impression. Il en est de même pour la lentille cendreuse $4 \mathrm{c}$, sur laquelle fut ensuite aménagé un radier de tessons. Elle montre la présence exclusive du buis (annexe 3) parmi ses charbons de bois, ce fait résultant d'un ramassage sélectif par l'homme dans un but restant à déterminer. La première phase de comblement de cette fosse (6-5$4 \mathrm{~d}-4 \mathrm{c}$ cendreux) découlerait donc d'une utilisation secondaire comme dépotoir au Bronze final 2 .

L'amas de tessons $4 c$, les lentilles de limons argileux $4 b$ et de cendres charbonneuses $4 a$ sont directement superposés suivant un plan circulaire grossier de $50 \mathrm{~cm}$ de diamètre, localisé dans la partie est de la fosse (fig. 4). Nous avons pu observer que les limons argileux $4 \mathrm{~b}$, de coloration orangée, étaient légèrement indurés. Leur contact direct avec les cendres de 4a ainsi que l'homogénéité et la densité de ces deux horizons tendraient à montrer qu'il y a eu combustion sur place. Par contre il est difficile de savoir quelle est la part qui revient au hasard ou à une volonté d'aménagement dans la présence successive des tessons de $4 \mathrm{c}$ et des limons $4 \mathrm{~b}$, bien que la cohérence de l'ensemble 4abc, tant sur le plan spatial que temporel, milite pour la seconde proposition. Par contre, aucun élément ne nous permet d'affirmer que les tessons contigus de $4 c$ aient été utilisés peu après
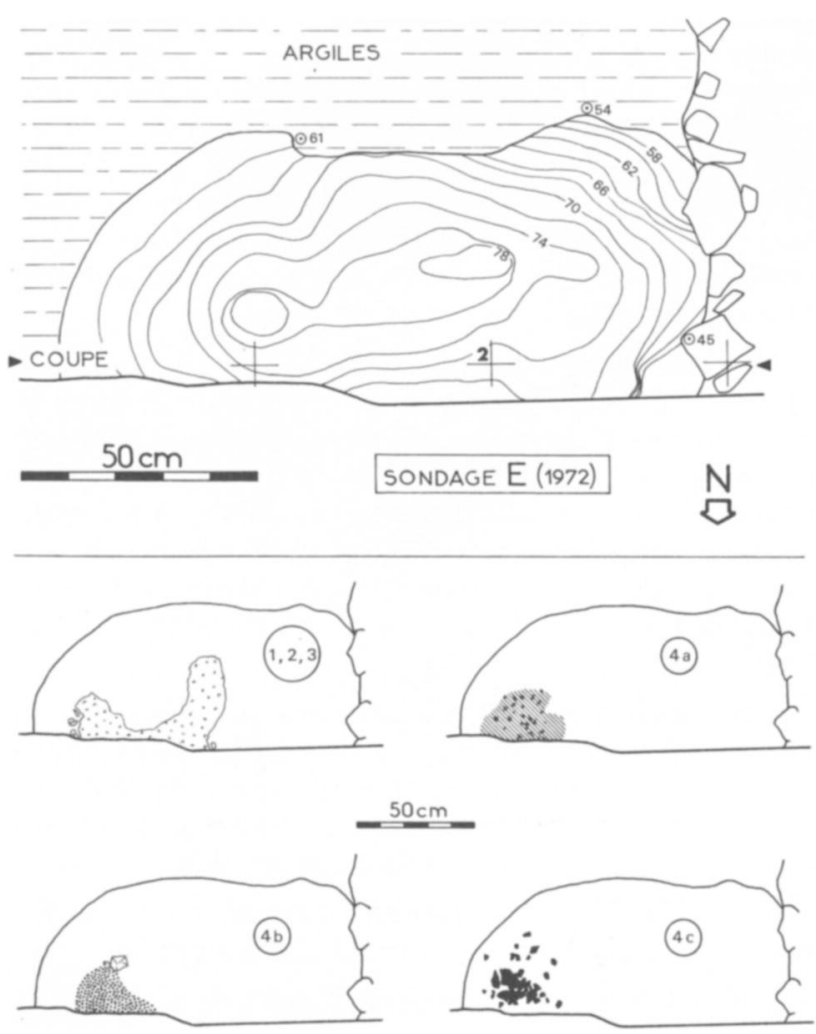

Fig. 4 - Plan en courbes de niveaux du fond de la structure et extension de quelques horizons sédimentaires naturels (couches $1,2,3$ ) et anthropiques (cendres $4 \mathrm{a}$, limons $4 \mathrm{~b}$ et radier de tessons $4 \mathrm{c}$ ).

leur abandon, ce qui nous empêche de fournir pour cet aménagement une datation équivalente à celle que nous suggère la typologie de ses céramiques, les données de $4 \mathrm{a}$ confortant cette position. Une seconde phase de comblement (4c-4b-4a) aurait donc pour origine la constitution et l'utilisation d'un petit foyer plat, à partir des vestiges et au sommet du dépotoir de la phase précédente. De semblables témoins de combustion constitués de radiers de tessons recouverts d'une chape d'argile indurée par le feu sont fréquents durant la Protohistoire. Plusieurs habitats proches en ont livré, notamment les cabanes de l'oppidum de La Liquière à Calvisson (Gard) et du site littoral de Tonnerre I (sondage 3) à Mauguio (Hérault) (Michelozzi, 1982, p. 31 et fig. 3, 4 et 11 ; Roudil, 1982, p. 457 ; Dedet, Prades, Py et SavayGuerraz, à paraitre) et il ne semble pas faire de doute que leur position centrée dans l'habitat les destinait à une fonction de cuisson (Michelozzi, 1982, p. 32). Un aménagement semblable a été décrit dans la grotte des Planches-près-Arbois (Pétrequin, 1981, fig. 229 et p. 214). Le faible volume des produits de combustion et la position latérale du foyer $4 \mathrm{abc}$, à l'entrée du couloir d'accès au réseau qui se développe dans l'obscurité, évoque aussi une fonction d'éclairage. De plus, l'observation des stratigraphies relevées au centre de la salle principale permet de 
penser que les foyers liés aux activités domestiques étaient avant tout établis dans ce secteur. La découverte de céréales carbonisées dans cette fosse rappelle l'association foyer-céramiques-céréales d'un des importants aménagements de l'occupation Bronze moyen de la proche cavité de la ChauveSouris (Vital, 1982), qui devait avoir été conçu pour cuire ou réchauffer le contenu de récipients, supposé être des préparations à base de céréales.

La couche 4 est composée de sédiments mêlés de blocs, de débris céramiques et osseux, de cendres et de charbons et les observations concernant le mode de dépôt de ces éléments sont de même nature que celles qui ont été faites sur les couches $4 c$ à 6 (phase 1). Cette troisième phase de remplissage résulterait du nettoyage d'un secteur occupé à la fin du Bronze final, la fosse fonctionnant à nouveau comme dépotoir.

Les couches 3 et 1 indiquent que plusieurs phases de concrétionnement dues à des infiltrations abondantes se sont déroulées, mais il reste difficile de les interpréter comme le résultat d'une augmentation du couvert végétal, de l'humidité, où les deux à la foi.

La couche 2 est le produit du remaniement des niveaux sous-jacents ou présents latéralement.

Les couches 3 et 1 marquent l'arrêt de la sédimentation détritique et anthropique témoignant des activités humaines dans cette partie de la cavité, et le changement radical de la dynamique sédimentaire.

Il reste à déterminer dans quel but cette excavation a été faite à l'origine. Si l'on rejette les hypothèses d'aménagement à fonction domestique de type foyer enterré ou silo pour la phase d'utilisation, l'interprétation comme fosse d'extraction de matériaux argileux au début du Bronze final est la plus vraisemblable.

\section{4. - LES INDUSTRIES}

\section{1. - Phase 1, couches 6-5-4d (fig. 5)}

Tous les fragments céramiques figurés appartiennent à des récipients bisegmentés et proviennent de la couche 5 .

Une forme non décorée à rebord divergent et point de segmentation médian $\left(n^{\circ} 4\right)$, de laquelle on peut rapprocher des rebords et une partie supérieure $\left(n^{\text {os }} 1\right.$ à 3 ), trouve des comparaisons fréquentes tant au Bronze final 2a qu'au Bronze final $2 b$ dans de nombreuses régions : nécropoles de Granges (Bonnamour, 1969, pl. 23, $\mathrm{n}^{\circ}$ 163) et des Gobillons à
Châtenay-sur-Seine (Bontillot, Mordant C., D. et Paris, 1975, fig. 11 et 14), scialet funéraire du Bois des Vouillants à Fontaine (Bocquet, 1963, fig. 4, $n^{\circ} 12$ ), les rapprochements les plus nets étant à faire avec les vases des grottes de la vallée de l'Ardèche : grotte proche d'Ebbou à Vallon-Pont-d'Arc (Ayrolles et Combier, 1979-80, fig. 5 C), grottes de Peyroche II à Auriolles et des Conchettes à Grospierres (Roudil et Saumade, 1968, fig. 67 ; Demont et Gros, 1968, fig. 16).

Plusieurs fragments portent des décors cannelés horizontaux et verticaux $\left(\mathrm{n}^{\text {os }} 5\right.$ à 7$)$ tout à fait caractéristiques du début du Bronze final du nord-est de la France (Mordant C. et D., 1970 ; Unz, 1973 ; Pétrequin, 1982) et des Alpes (Bocquet, 1963, fig. 2), mais que l'on retrouve parfois durant tout le Bronze final 2 dans la Drôme et dans le Gard, dans les grottes de la Baume Sourde à Francillon (Cornet, 1963, fig. 5 à 7) et du Prével Supérieur à Montclus (Roudil, 1972, fig. 62), accompagnés de coupes à décor incisé interne. C'est également vers les contextes régionaux que renvoient les tessons à décor pincé digité $\left(\mathrm{n}^{\circ} 8\right)$ et pincé impressionné à l'ongle $\left(n^{\circ} 9\right)$. Le premier s'identifie au fragment de partie supérieure décorée de la couche $4 \mathrm{c}$ (fig. $6, \mathrm{n}^{\circ} 20$ ), connu en Languedoc oriental dans les grottes des Cloches à Saint-Martin-d'Ardèche et du Prével Supérieur (Roudil, 1972, fig. $74 \mathrm{n}^{\circ} 10$ et pl. 19 et 20) en contexte Bronze final 2 . Le décor vertical pincé est bien représenté dans la Drôme et en Ardèche dans les grottes d'Antonnaire à Montmaur et du Pontiar à Vallon-Pont-d'Arc (3), ainsi que dans la grotte de Peyroche II sur une forme que l'on peut dater, comme les précédentes, du Bronze final 1-2 (Roudil et Saumade, 1968, fig. 70). Deux vases issus des fouilles anciennes de la Baume des Anges sont décorés d'un motif semblable réalisé à la cordelette dans un cas, et suivant la même technique que le tesson décrit ici dans le second cas, ce qui démontre que cette dernière est en fait une imitation du motif cordé (Vital, 1984, p. 215). Un dernier fragment provient d'un vase bisegmenté bas décoré de fines cannelures horizontales $\left(n^{\circ} 10\right)$ dont nous connaissons de nombreux exemples dans le sud-est au Bronze final 2a : couche 3 de la grotte de Labeil à Lauroux (Bousquet, Gourdiole et Guiraud, 1966, fig. 18), grottes du Pontiar et de Peyroche II (Roudil et Saumade, 1968, fig. 88). Ils sont plus rares en Provence, ainsi qu'en dehors du Bassin rhodanien, seule la grotte des Fées à Châteauvieux semble en avoir livré (Lagrand, 1968, pl. 19, n 2 ).

L'industrie en silex est composée d'un burin sur cassure $\left(\mathrm{n}^{\circ} 12\right)$ et d'une armature triangulaire à base convexe $\left(n^{\circ} 11\right)$. Il est peu aisé de savoir si ces objets sont synchrones des céramiques, bien que l'homogé

(3) Ce mobilier issu des fouilles réalisées par A. Héritier est en cours d'étude par nos soins. 


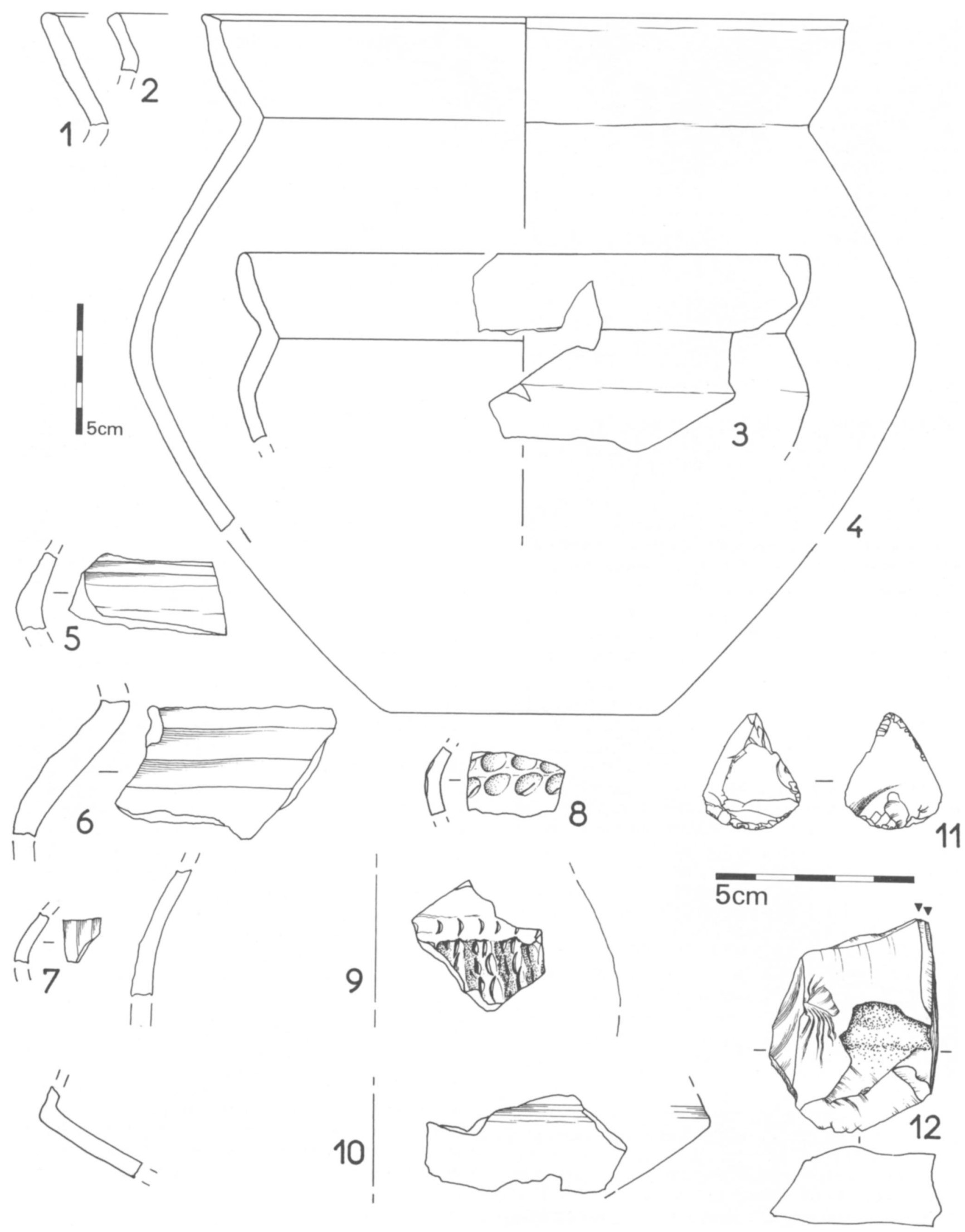

Fig. 5 - Industries céramique et lithique de la couche 5. 


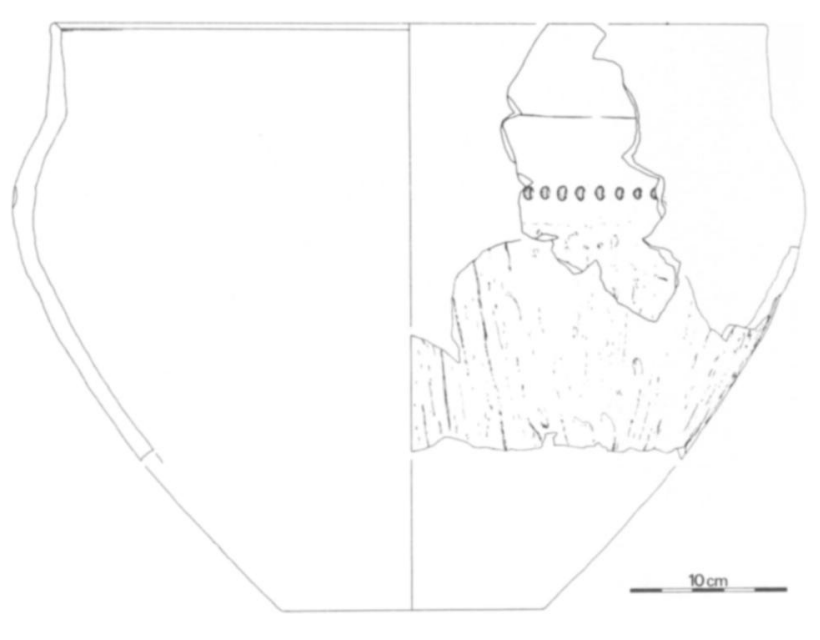

néité chronologique de ces dernières plaide en ce sens, car ces deux pièces peuvent tout aussi bien provenir du remaniement et du rejet de sédiments des niveaux du Néolithique final de la salle principale. Néanmoins, des armatures en silex sont connues en milieux Bronze final indiscutables sur les sites helvétique et germanique de Bavois-en-Raillon (Vital et Voruz, 1984, fig. $56 \mathrm{n}^{\text {os }} 45$ et 46 ) et de Neckarsulm (Dehn, 1972, pl. 25, n 34).

\section{2. - Phase 2, couches $4 c-4 b-4 a$ (fig. 6 et 7 )}

La seule forme complète connue (fig. 6) a servi d'assise à la structure de combustion de la phase 2. Sa partie inférieure est raclée et s'articule sur un point de segmentation souligné par une ligne horizontale d'impressions digitées ; son rebord est vertical. Les ensembles de Pommiers-en-Forez et de l'aven de la Sessa à Lagorce renferment des récipients comparables en milieu Bronze final 2 évolué (Vaginay et al., 1982 , fig. $7, \mathrm{n}^{\text {os }} 1$ et 2 ; Roudil, 1963, fig. 3 n $^{\circ} 3$ ). Une datation similaire doit être retenue pour les fragments de rebords divergents non décorés (fig. $7, \mathrm{n}^{\text {s }}$ 13 et 15 à 17$)$ et digité $\left(n^{\circ} 14\right)$, ainsi que pour les bords de coupes divergentes qui font leur apparition en $4 c\left(n^{\text {os }} 10\right.$ à 12$)$ et qui abondent dans les sites de la fin du Bronze final 2.

La seule pièce réalisée en matériau non céramique est une fusaïole ( $\left.n^{\circ} 21\right)$ tirée d'une roche calcaire.

Les nombreux raccords et similitudes entre tessons des couches $4 c$ et 5 , dont le fragment $n^{\circ} 19$ est un témoin, démontrent que la subdivision stratigraphique entre $4 c$ et 5 ne recouvre peut-être pas une réalité chronologique ; d'ailleurs, autant dans l'une ou l'autre de ces couches, les éléments céramiques peuvent être rapportés à chacun des deux stades du Bronze final 2. Ce phénomène n'est pas isolé puisqu'il en est de même de plusieurs éléments des grottes du Prével Supérieur et de la Baume Sourde, ce qui nous a récemment conduit à poser l'hypothèse de l'utilisation, dans le Défilé de Donzère, d'un certain nombre de types céramiques tout au long du Bronze final 2, les apports que l'on a coutume d'attribuer au groupe Rhin-Suisse étant mineurs (Vital, 1984, pp. 299-300). Si ces apports sont particulièrement nets dans le Bassin parisien où il est même question de migrants venus de l'Est qui se singularisent par l'utilisation exclusive de l'incinération comme mode funéraire (Mordant, 1981 et 1982), comme en Franche-Comté où l'évolution des industries et des habitats se traduit par un renouvellement partiel de la population à partir du Bronze final $2 \mathrm{~b}$ (Pétrequin, 1982), il faut considérer que ce mouvement doit être atténué dans nos régions, sur la base des observations énoncées précédemment.

Les deux tessons de la couche cendreuse $4 \mathrm{a}$ ( $\mathrm{n}^{\text {os }} 8$ et 9) sont à rattacher à une phase plus tardive du Bronze final. Le premier rappelle les découvertes faites récemment dans la grotte de la Chauve-Souris dont les couches $8 \mathrm{c} / 9$ ont livré plusieurs récipients complets de morphologie semblable en milieu Bronze final 3b (Vital, 1984, pl. 151, $\mathrm{n}^{\circ} 2$ et 157 , $\mathrm{n}^{\text {os }} 10$ à 12). Ces données sont les seules qui pourraient orienter la datation de la structure 4abc vers le Bronze final 3, confirmant dans ce cas l'utilisation secondaire des tessons BF 2 du radier $4 c$, bien que l'unité du dispositif puisse plaider en faveur d'une datation contemporaine du récipient de $4 c$.

N'oublions pas néanmoins que si les assemblages typologiques des phases de remplissage de la fosse montrent une cohérence certaine, ceux-ci résultent de rejets secondaires et ne présentent pas les meilleures garanties d'homogénéité.

\section{3. - Phase 3, couche 4 (fig. 7)}

Hormis deux rebords divergents peu caractéristiques ( $\mathrm{n}^{\text {os }} 23$ et 24 ) et un tesson portant un téton, surement issu des niveaux inférieurs $\left(n^{\circ} 29\right)$, deux formes monosegmentées à partie supérieure convergente et un fragment à décor de doubles cannelures horizontales peuvent se prêter au jeu des comparaisons $\left(\mathrm{n}^{\text {os }} 26\right.$ à 28$)$. Le premier a été rencontré dans les palafittes d'Auvernier et du lac du Bourget (Rychner, 1979, pl. 19, no 10 ; Coutil, 1915, pl. III, $\left.\mathrm{n}^{\circ} 16\right)$ et dans le faciès Mailhac 1 en Languedoc, dans la couche $4 \mathrm{du}$ sondage 1 de l'oppidum du Roc de Gachonne à Calvisson (Garmy, 1979a, fig. 10, n 169) 

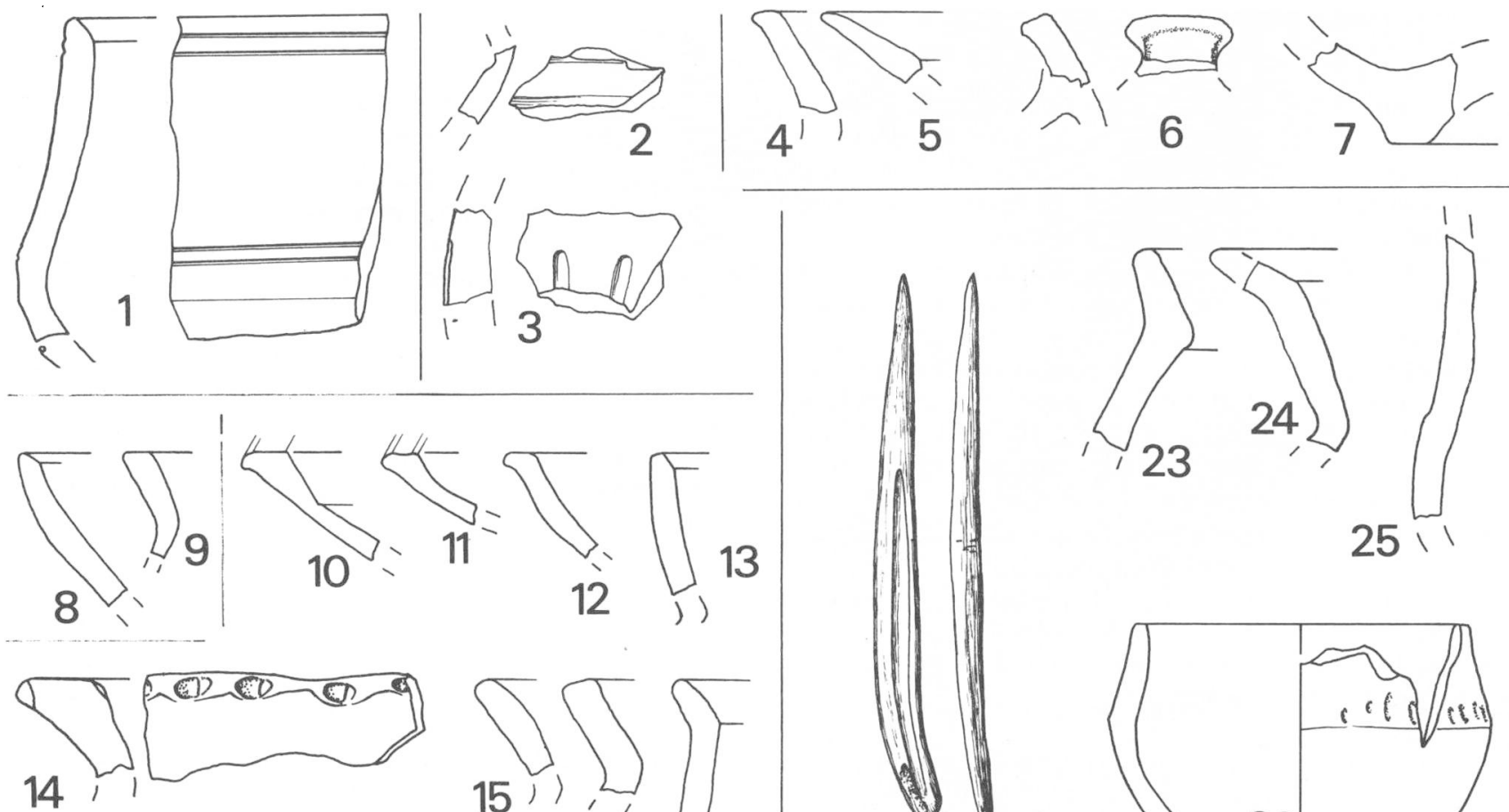

14

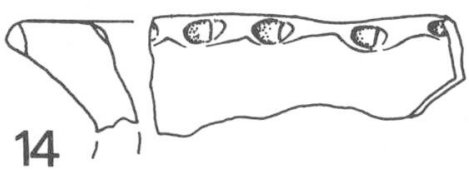

$10 \mathrm{~cm}$
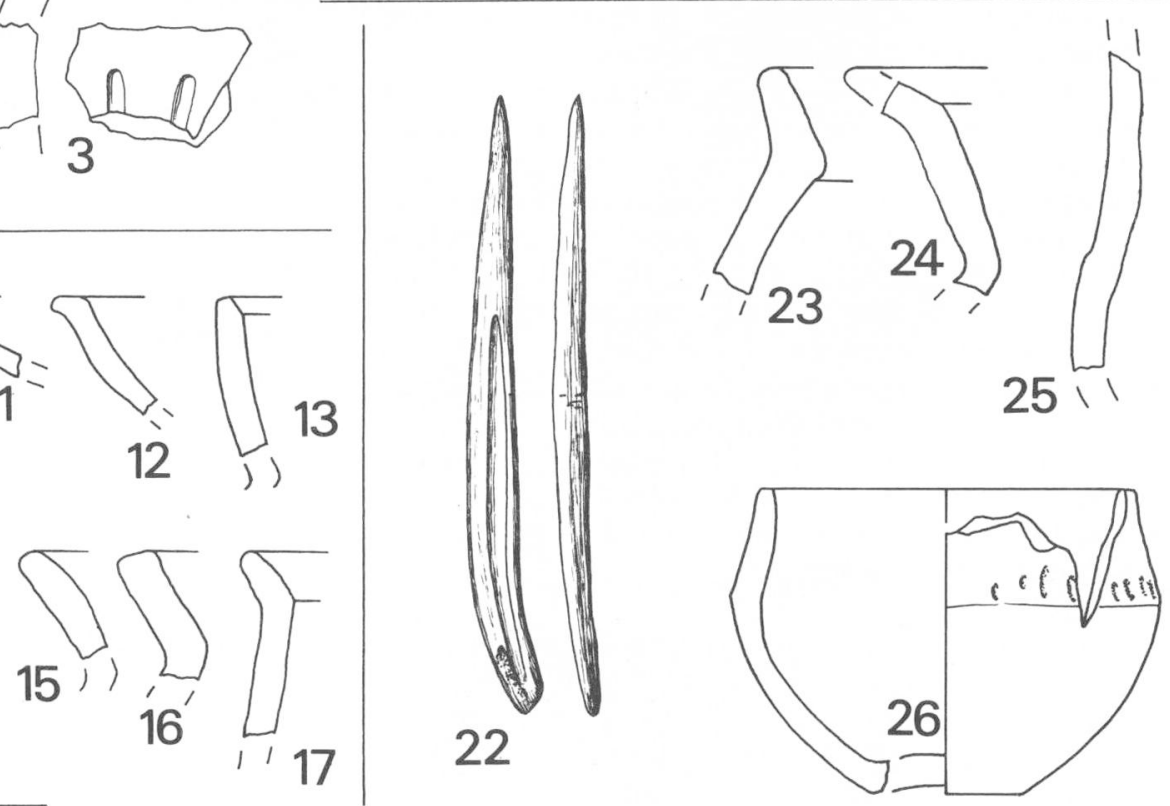

12 )

13
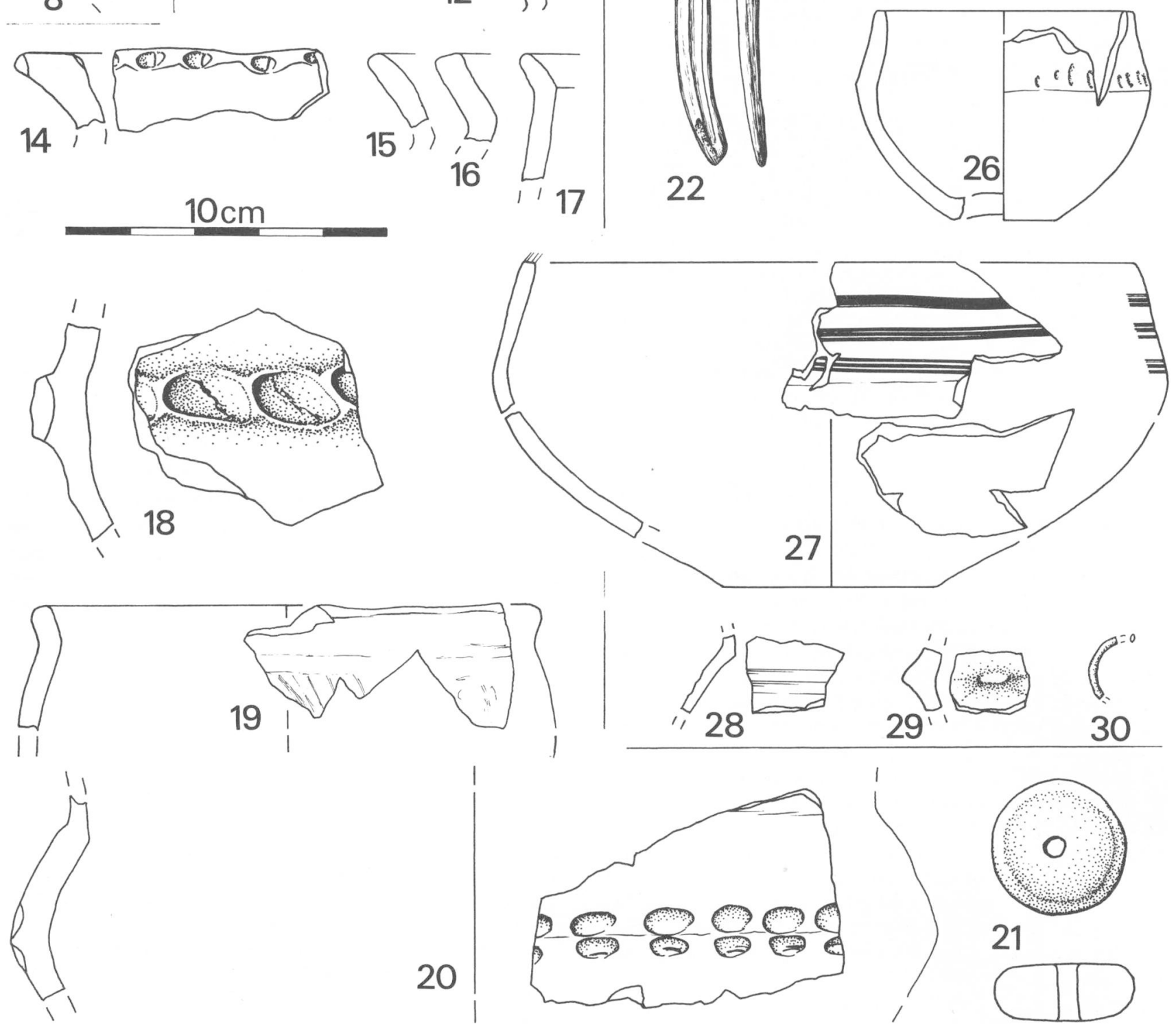

Fig. 7 - Industries céramique, lithique, osseus

couches $4 c$ et $5 ; 22$ à 30 : couche 4 . 
et avec un décor caractéristique de ce groupe dans la tombe 68 de la nécropole du Moulin de Mailhac (Louis et Taffanel O. et J., 1958, fig. 9). Bien que d'encombrement supérieur, un vase de la bergerie Hermet à Calvisson peut lui être comparé (Garmy, $1979 \mathrm{~b}$, fig. $\left.7, \mathrm{n}^{\circ} 159\right)$. Les trouvailles ardéchoises et drômoises sont représentées par la tombe de faciès mailhacien - rasoir à manche et pince à épiler - du tumulus $\mathrm{n}^{\circ} 1$ de l'Abeillou à Grospierres (Gros O. et A.-C., 1972, fig. 4) et par la couche F de l'oppidum du Pègue (Lagrand et Thalmann, 1973, pl. I, n $\left.{ }^{\circ} 17\right)$. Toutes ces découvertes se classent au Bronze final $3 b$. La seconde forme $\left(n^{\circ} 27\right)$ se rattache au faciès Mailhac 1 dont l'oppidum de Roque de Viou à SaintDionisy a fourni de bons exemples (Garmy, 1974, fig. 5 ; Garmy et Py, 1976, fig. 16), mais elle est aussi connue sur les sites évoqués précédemment, tout comme le tesson à décor cannelé $\left(n^{\circ} 28\right)$ appartenant à une petite forme subsphérique.

L'industrie non céramique comprend un anneau de bronze $\left(\mathrm{n}^{\circ} 30\right)$ et une pointe en os à base déjetée $\left(\mathrm{n}^{\circ}\right.$ 22). Elle est tirée d'une diaphyse de grand ruminant et a été façonnée par raclage ; elle montre dans sa partie médiane de fines stries transversales postérieures à sa fabrication. Ce type d'objet est très répandu sur les sites néolithiques littoraux des lacs de Suisse occidentale. Il s'apparente à celui de SaintAubin (Neuchâtel) qui avait conservé sa hampe de bois (de Mortillet, 1890, fig. 48) et peut être interprété comme engin de pêche ou de chasse. Comme le nôtre, les éxemplaires néolithiques montrent souvent de fines stries transversales à l'emplacement de la ligature.

Concernant la couche 4 de la fosse de la Baume des Anges, nous avons pu constater que les types céramiques mailhaciens 1 du Bronze final $3 \mathrm{~b}$ étudiés ici étaient inconnus dans l'ensemble $8 \mathrm{c} / 9$ de la grotte de la Chauve-Souris (Vital, 1984, pp. 302-303). Ce dernier renferme de surcroît des formes qui évoquent celles du Premier âge de Fer languedocien des tumulus des Garrigues (Gasco, 1978, fig. 4) et de la période II de la Vaunage, bien attestée sur l'oppidum de La Liquière à Calvisson (Dedet et Py, 1976). Cette observation, ainsi que des comparaisons avec les éléments d'un Bronze final $3 b$ tardif de l'oppidum de La Redoute à Beaucaire (couche 5 du sondage 2 ; Dedet, Michelozzi, Py, Raynaud et Tendille, 1978) nous permettent d'interpréter cette différence en terme chronologique, l'occupation de la couche 4 de la Baume des Anges précédant celle de la grotte de la Chauve-Souris. Ce fait confirme les conclusions auxquelles sont parvenus les chercheurs languedociens (op. cit. ; Dedet, 1978 et 1981 ; Garmy, 1979a et c ; Py et Raynaud, 1982) concernant l'existence d'une phase prenant place entre le Bronze final $3 \mathrm{~b}$ du genre Mailhac 1 et le faciès "suspendien » (de la grotte Suspendue à Collias) du Premier âge du Fer.

\section{4. - Phase 4, couches 3 à 1 (fig. 7)}

Le mobilier hétérogène issu des couches 1 ( $\mathrm{n}^{\text {os }} 2$ et 3) et $2\left(n^{\text {os }} 4\right.$ à 7$)$ date de plusieurs stades du Bronze final, tessons à décors cannelés, fond plat à dépression, anse "ad ascia », ce qui démontre que les sédiments de ces niveaux résultent en partie du remaniement de formations antérieures.

Parmi les éléments découverts hors stratigraphie, un fragment de partie supérieure décorée de doubles incisions surmontant un point de segmentation souligné par des méplats se rattache aux productions du Bronze final $3 b\left(n^{\circ} 1\right)$.

\section{5. - CONCLUSION}

La fosse décrite ici a connu aux moins deux utilisations : comme dépotoir et comme réceptacle d'une structure de combustion, au Bronze final. Les diagrammes anthracologiques indiquent que les essences représentées dans le foyer $4 \mathrm{a}$ et dans les rejets $4 \mathrm{c}$ diffèrent notablement, la présence exclusive du Buis en $4 c$ témoignant d'une activité particulière. A titre d'hypothèse plusieurs fonctions peuvent être évoquées : besoin d'un combustible lent, fumigation, cuisson ou traitement restant à préciser appliqué à des céramiques... Dans ce dernier cas, et si l'on veut lui accorder quelque crédit, le façonnage des poteries a pu déterminer et suivre l'extraction des sédiments de la fosse ; une expérimentation devrait permettre de conforter ou de rejeter cette explication globale.

Cette étude a démontré l'intérêt de la fouille des derniers témoins de remplissage de la Baume des Anges et des opérations similaires seront conduites dans d'autres sites majeurs fouillés anciennement dans la moyenne vallée du Rhône. Nous pourrons ainsi aborder le reclassement des importantes séries ardéchoises et drômoises en nous appuyant sur des ensembles de référence locaux, ce qui permet d'atténuer les erreurs de sériations engendrées parfois par des comparaisons à trop longue distance.

Institut du Quaternaire,
Université de Bordeaux I, 33405 Talence Cedex

\footnotetext{
Ayrolles P. et Combier J. (1979-80) - Un nouveau musée régional de Préhistoire. Études Préhistoriques, n ${ }^{\circ} 15$, pp. 49-55.

BocQuet A. (1963) - Le scialet funéraire du Bois des Vouillants-Fontaine (Isère). Bull. de la Soc. préhist. franç., t. LX, pp. 847-867.

Bonnamour L. (1969) - L'âge du Bronze au musée de Chalonsur-Saône. Chalon-sur-Saône, 94 p., XXXIII pl.
} 
Bontillot J., Mordant C., D. et Paris J. (1975) - La nécropole des Gobillons à Châtenay-sur-Seine (Seine-et-Marne). Bull. de la Soc. préhist. franç., t. 72, pp. 416-456.

Bornand M., Combier J. Lorenchet de MonTJamont M. et al. (1979) - Carte géologique de la France à 1/50 000, Montélimar, $n^{o}$ 866. Ed. du B.R.G.M., Orléans, 35 p.

Bousquet N., Gourdiole R. et Guiraud R. (1966) - La grotte de Labeil, près de Lauroux (Hérault). Cahiers ligures de Préhist. et d'Archéol., t. 15, pp. 79-166.

Coutil L. (1915) - La céramique des palafittes du Lac du Bourget. Bull. de la Soc. préhist. franç., t. XII, pp. 386-431.

Debelmas J. (1974) - Géologie de la France. 2. Les chaînes plissées du cycle alpin et leurs avant-pays. Éd. Doin, Paris, 248 p.

DEDET B. (1978) - L'habitat de hauteur du Grand-Ranc à Boucoiran (Gard) et le Bronze final IIIb dans les garrigues du Languedoc oriental. Gallia Préhistoire, t. 21, fasc. 1, pp. 189-206.

DEDET B. (1981) - Le gisement protohistorique de la combe de Montaillon à Sanilhac-et-Sagriès (Gard). Doc. d'Archéol. méridionale, $\mathrm{n}^{\circ} 4$, pp. 51-59.

Dedet B., Michelozzi A., Py M., Raynaud C. et Tendille C. (1978) - Ugernum, Protohistoire de Beaucaire. Cahier $\mathrm{n}^{\circ} 6 \mathrm{de}$ l'A.R.A.L.O., Caveirac, 156 p., 87 fig.

Dedet B., Prades H., Py M. et Savay-Guerraz H. L'occupation des rivages de l'Étang de Mauguio (Hérault) au Bronze final et au Premier âge du Fer. Manuscrit polycopié, 495 p. et 286 fig., à paraître.

DEHN R. (1972) - Die Urnenfelderkultur in Nordwürttemberg. Forschungen und Berichte zur Vor- und Frühgeschichte in BadenWürttemberg, I, Müller und Gräff, Stuttgart, 136 p., 35 pl.

Demarco G. (1973) - Lyonnais. Vallée du Rhône. Guides géologiques régionaux, Éd. Masson et Cie, Paris, $175 \mathrm{p}$.

DemonT J. et Gros A.-C. (1968) - Un important habitat préhistorique à Grospierres (Ardèche) : la grotte des Conchettes Grou Peïro, no 1 , pp. 17-24.

GARMY P. (1979a) - Premières recherches sur l'oppidum du Roc de Gachonne à Calvisson (Gard). Archéol. en Languedoc, $\mathrm{n}^{\circ}$ 2, pp. 97-114.

GARMY P. (1979b) - Un village du Bronze final IIIb à la Bergerie Hermet (Calvisson, Gard). Doc. d'Archéol. méridionale, $\mathrm{n}^{\circ}$ 2, pp. 5-15.

GARMY P. (1979c) - L'évolution de l'Âge du Bronze Final IIIb et la transition Bronze/Fer en Languedoc Oriental d'après la culture matérielle des habitats. État des questions. Dialogues d'Histoire Ancienne, 5, Ann. Litt. de l'Univ. de Besançon, n 239 , pp. 23-34.

GARMy P. et Py M. (1976) - Deux cabanes stratifiées dẹ l'Ầge 'du Bronze final IIIb sur l'oppidum de Roque de Viou à SaintDionisy (Gard). Gallia Préhistoire, t. 19, fasc. 1, pp. 239-259.

Gasco Y. (1978) - Les tumulus du Premier âge du Fer en Languedoc Oriental. Thèse de III $^{e}$ cycle, Université Paul Valéry, Montpellier, 463 p., 107 pl.

Gros O. et A.-C. (1972) - Le tumulus protohistorique $\mathrm{n}^{\circ} 1$ de l'Abeillou à Grospierres. Études Préhistoriques, no 2, pp. 15-19.

LAGRAND C. (1968) - Recherches sur le Bronze final en Provence méridionale. Thèse de l'Université d'Aix-en-Provence, 394 p., LXXXVIII pl.

LagRand C. et Thalmann J.-P. (1973) - Les habitats protohistoriques du Pègue (Drôme). Le sondage $\mathrm{n}^{\circ} 8$. Cahier $\mathrm{n}^{\circ} 2 \mathrm{du}$ C.D.P.A., Grenoble, 159 p., XXXIX pl.

Lambert M. (1980) - Le Défilé de Donzère. Rev. Drômoise, t. LXXXVIII, ${ }^{\circ} 415$, pp. 21-30.
LAMBERT M. et Coll. (1976) - Les grottes de Donzère. In : Livret-guide de l'excursion A9: Néolithique det Âges des Métaux dans les Alpes françaises. IX ${ }^{\mathrm{e}}$ Congrès de l'U.I.S.P.P., Nice, pp. 32-39.

Louis M. et Taffanel O. et J. (1958) - Le Premier âge du Fer languedocien. Institut international d'études ligures, Bordighera, Montpellier. II : les nécropoles à incinérations, 264 p., 213 fig.

MiCHELOZZI A. (1982) - L'habitation protohistorique en Languedoc oriental. Cahier $\mathrm{n}^{\circ} 10$ de l'A.R.A.L.O., Caveirac, 95 p., 45 fig.

Mordant C. (1981) - Les enclos de l'Âge du Bronze du confluent Seine-Yonne. In : Enclos funéraires et structures d'habitat en Europe du Nord-Ouest. Trav. du Lab. Antropologie, Préhistoire, Protohistoire, Quaternaire armoricains, Rennes, (1983), pp. 163-180.

Mordant C. (1982) - L’Âge du Bronze dans le Sénonais. Introduction. In : Préhistoire du Sénonais, Sens, pp. 105-110.

MoRDANT C. et D. (1970) - Le site protohistorique des Goursaux-Lions à Marolles-sur-Seine (Seine-et-Marne). Mém. de la Soc. préhist. franç., t. 8, Éd. Klincksieck, Paris, 139 p., 66 fig.

MORTILlet G. de (1980) - Des origines de la chasse, de la pêche et de l'agriculture. Tome I, chasse, pêche, domestication. Éd. Lecrosnier et Babé, Paris, 512 p., 148 fig.

Petrequin P. (1981) - La grotte des Planches-près-Arbois (Jura). Proto-Cortaillod et Âge du Bronze final. Thèse de Doctorat d'État, Faculté des Lettres de Besançon, 379 p., 333 fig., à paraître.

Petrequin P. (1982) - Die jüngere Bronzezeit im französischen Jura. Archäologisches Korrespondenzblatt, t. 12, 2, pp. 167178.

Py M. et Raynaud C. (1982) - Stratigraphie du Marduel (Saint-Bonnet-du-Gard). Doc. d'Archéol. méridionale, $\mathrm{n}^{\circ} 5$, pp. 5-32.

Roudil J.-L. (1963) - Dépôt des champs d'urnes du plateau de Lagorce (Ardèche). Gallia Préhistoire, t. VI, pp. 129-132.

RoudiL J.-L. (1972) - L'Âge du Bronze en Languedoc oriental. Mém. de la Soc. préhist. franç., t. 10, Éd. Klincksieck, Paris, 302 p., 108 fig.

RoudiL J.-L. (1982) - Informations archéologiques. Circonscription de Languedoc-Roussillon. Gallia Préhistoire, t. 25, fasc. 2, pp. 437-468.

Roudil J.-L. et Saumade H. (1968) - La grotte de Peyroche II à Auriolles (Ardèche). Gallia Préhistoire, t. XI, fasc. 1, pp. 147203.

RYCHNER V. (1979) - L'Âge du Bronze final à Auvernier. Typologie et chronologie des anciennes collections conservées en Suisse. Cahiers d'Archéol. Romande de la Bibliothèque Historique Vaudoise, Lausanne, $\mathrm{n}^{\text {os }} 15$ et 16,166 p. et $324 \mathrm{p}$.

UNZ C. (1973) - Die spätbronzezeitliche Keramik in Südwestdeutschland, in der Schweiz und in Ostfrankreich. Praehistorische Zeitschrift, t. 48, 1, pp. 1-123.

VAGinay M. et al. (1982) - L'Âge du Bronze dans la Loire à la lumière des fouilles récentes. Cahiers archéol. de la Loire, $\mathrm{n}^{\circ} 2$, pp. 17-37.

ViTAL J. (1982) - Recherches sur l'Âge du Bronze dans le Défilé de Donzère (Drộme). Nouv. arch. Mus. Hist. nat. de Lyon, fasc. 20, suppl., pp. 57-61.

VITAL J. (1984) - Sites et industries de l'Âge du Bronze dans le Défilé de Donzère (Drôme). Thèse de $3^{\mathrm{e}}$ cycle de l'Université de Bordeaux I, Géologie du Quaternaire et Préhistoire, nº 1957, 386 p., $166 \mathrm{pl}$.

VITAL J. et VORUZ J.-L. (1984) - L'habitat protohistorique de Bavois-en-Raillon (Vaud). Cahiers d'Archéol. Romande de la Bibliothèque Historique Vaudoise, Lausanne, $n^{\circ} 28,231 \mathrm{p}$. et 115 fig. 
Annexe 1

\title{
La faune de la Baume des Anges (Drôme, France) (Bronze final)

\author{
par Louis Chaix
}

La zone de rejet des vestiges attribuables soit au Bronze final 2, soit au Bronze final 3b a livré de rares vestiges osseux animaux. Ceux-ci sont au nombre de 114 en regroupant les divers niveaux. Ce nombre est trop faible pour permettre des comparaisons externes ou même pour étudier d'éventuelles variations entre deux périodes. Nous nous contenterons donc de donner les résultats bruts, assortis de quelques rares commentaires.

Le tableau suivant résume la répartition chronologique des vestiges.

\begin{tabular}{|c|c|c|c|c|}
\hline Espèce & $\begin{array}{l}\text { Couche } \\
5 \\
\text { B.F. } 2\end{array}$ & $\begin{array}{l}\text { Couche } \\
4 \mathrm{c} \\
\text { B.F. } 2\end{array}$ & $\begin{array}{c}\text { Couche } \\
4 \mathrm{a} \\
\text { B.F. }\end{array}$ & $\begin{array}{l}\text { Couche } \\
4 \\
\text { B.F. } 3 b\end{array}$ \\
\hline $\begin{array}{l}\text { Caprinés (Ovis/Capra) } \\
\text { Bœuf (Bos taurus L.) } \\
\text { Porc (Sus domesticus } \mathrm{Br} .)\end{array}$ & $\begin{array}{l}4 \\
2\end{array}$ & 4 & 一 & $\begin{array}{l}8 \\
1 \\
2\end{array}$ \\
\hline $\begin{array}{l}\text { Cerf (Cervus elaphus L.) } \\
\text { Sanglier (Sus scrofa L.) } \\
\text { Lapin (Oryctolagus } \\
\text { cuniculus L.) } \\
\text { Castor (Castor fiber L.) } \\
\text { Suidés (porc/sanglier) } \\
\text { Chiroptères }\end{array}$ & 4 & 1 & 11 & $\begin{array}{l}3 \\
6 \\
4 \\
7 \\
2\end{array}$ \\
\hline $\begin{array}{l}\text { Côtes } \\
\text { Vertèbres }\end{array}$ & & & 3 & $\begin{array}{l}8 \\
8\end{array}$ \\
\hline Indéterminés & 5 & 2 & 1 & 39 \\
\hline Total & 11 & 10 & 6 & 97 \\
\hline
\end{tabular}

Les animaux domestiques semblent dominés par les caprinés. On pouvait du reste s'y attendre dans cette zone géographique. Parmi eux nous avons mis en évidence la présence du mouton (Ovis aries L.) et de la chèvre (Capra hircus L.).

Un radius de mouton nous a donné une taille au garrot de 59,3 cm (Teichert, 1975) ce qui est plutôt faible (Chaix, 1977).

(1) Ce travail a pu être réalisé grâce au concours du Fonds National Suisse de la Recherche Scientifique (Requête $n^{\circ} 1580-0.82$ ) que nous remercions ici.
Du point de vue de l'âge des caprinés les rares vestiges observés montrent la présence d'au moins un animal très jeune et d'un autre individu de moins de 3 ans et demi.

Certains ossements montrent des traces de boucherie, par exemple un talus droit d'agneau.

Nous donnons ici les rares mesures que nous avons pu prendre sur les restes des caprinés :

Ovis aries L. (couche 5)

$\begin{array}{llr}\text { Radius } & \text { Long. tot. } & 147.6 \\ & \text { DT pm. } & 30.1 \\ & \text { DT min. dia. } & 15.0 \\ \text { DT dm. } & 28.7\end{array}$

Ovis aries L. (couche 4)

Humérus DT dm. 27.5

Capra hircus L. (couche 4)

$\begin{array}{lll}\text { Talus } & \text { L. ext. } & 27.5 \\ & \text { L. int. } & 25.9 \\ & \text { DT tête } & 18.4\end{array}$

Le porc (Sus domesticus Br.) est mal représenté.

Il est fort probable que les quelques vestiges non déterminés spécifiquement de la couche 4 appartiennent à des animaux domestiques. La présence d'une jeune femelle est attestée par un fragment de maxillaire droit. Les autres vestiges montrent l'existence d'un individu de moins de 3 ans.

Une seconde phalange (couche 5) nous a donné les mesures suivantes :

L.

DT pm. $\quad 14.8$

DT min. dia. $\quad 12.2$

DT dm. $\quad 12.1$

Parmi les animaux sauvages, il faut signaler la présence du cerf élaphe. Certains ossements (couches 4 et $4 c$ ) portent des traces de découpe ou des coups.

Les mesures prises semblent indiquer des individus de petite taille (Pietschmann, 1977). 
\begin{tabular}{lllll} 
Humérus & couche 4c) & Patella & \multicolumn{2}{c}{ Os malléolaire } \\
DT dm. & 54.0 & Haut. 46.0 & DAP & 24.8 \\
& & DT 37.1 & &
\end{tabular}

Phalange 1 (couche 4) Phalange 3 (couche 4)

DT pm.

21.6 L. diagonale

53.7

Le sanglier est attesté par 3 ossements appartenant à un jeune et à un adulte. Un fragment proximal de phalange 1 nous a donné un DT pm. de $23.7 \mathrm{~mm}$ ce qui représente un individu très robuste, comparable aux gros mâles du néolithique d'Europe occidentale.

Le lapin (Oryctolagus cuniculus L.) est également présent dans les couches $4 a$ et 4 .

Les quelques mesures prises sont très proches des moyennes observées sur des lapins espagnols de l'âge du Bronze (von den Driesch et Boessneck, 1970).

$\begin{array}{llr}\text { Scapula } & \text { DAP min. col. } & 4.3 \\ & \text { DT cavité glén. } & 7.2 \\ & \text { DAP proc. artic. } & 8.3 \\ \text { Ulna } & \text { Long. tot. } & 71.5\end{array}$

Le lapin donne une touche méridionale à cette faune. Il est fréquent en effet dans de nombreux sites du sud et du sud-ouest de la France, alors qu'il est très rare ou absent au nord (Donard, 1982).

La proximité du Rhône est enfin indiquée par la présence de plusieurs ossements de castor (Castor fiber L.), essentiellement dans la couche 4. Ces restes sont attribuables à un individu adulte.

Nous donnons ici les quelques données métriques prises :

$\begin{array}{llr}\text { Fémur } & \text { Long. depuis la tête } & 119.5 \\ & \text { Long. max. } & 125.5 \\ & \text { DT pm } & 47.8 \\ & \text { DT dm } & 44.2 \\ \text { Talus } & \text { Long. } & 22.9\end{array}$

Les mesures du fémur indiquent un animal de forte taille, comparable aux plus gros spécimens du néolithique d'Europe occidentale (Boessneck et al., 1963).

Nous n'avons pas relevé de traces de boucherie, mais un fragment de temporal pourrait indiquer le bris d'un crâne, assez solide chez cet animal.

La couche 4 enfin a livré deux ossements de chiroptères. Il s'agit de l'extrémité proximale d'un radius gauche de grand rhinolophe fer-à-cheval $(R h i-$ nolophus ferrumequinum Schreb.) (2) et d'un métacarpien spécifiquement indéterminé.

A ces divers ossements, il faut ajouter un certain nombre de vertèbres et de côtes non attribuées spécifiquement. Disons simplement qu'elles semblent se répartir en deux groupes : des pièces de taille

(2) Nous remercions le Prof. V. Aellen de cette détermination. moyenne à rattacher au cerf et aux suidés et d'autres, plus petites, de la taille des caprinés.

Plusieurs fragments enfin n'ont pu être attribués. Certains d'entre eux montrent des traces de carbonisation et quelques rares stries de décarnisation.

Pour conclure cette courte étude, nous devons tout d'abord constater la très grande faiblesse numérique de l'échantillon qui ne nous permet aucune comparaison entre les niveaux du Bronze final 2 et le B.F. 3b.

Quelques remarques peuvent être faites sur l'ensemble de la faune. Les espèces chassées sont assez bien représentées, alors que pour la plupart des sites de cette époque, ce sont les animaux domestiques qui l'emportent très nettement (Borrello et Chaix, 1983 ; Poulain, 1964). On peut aussi noter l'absence du chien et du cheval, assez fréquents dans d'autres sites.

Cette courte présentation demandera à être complétée par des études d'échantillons plus importants, permettant une meilleure vision du monde animal et de ses relations avec les hommes du Bronze final dans la Drôme.
Boessneck J., Jequiert J.-P. et Stampfli H.-R. (1963) Seeberg-Burgäschisee-Süd. Teil 3 : Die Tierreste, Acta Bernensia II, Bern, Stämpfli et Cie.

Borrello M. et Chaix L. (1983) - Étude de la faune de Hauterive-Champréveyres (Neuchâtel), (Bronze final), (19791980), Bull. Soc. Neuchât. Sci. Nat., 106, pp. 159-169.

ChaIX L. (1977) - Les moutons préhistoriques de la haute vallée du Rhône, Ethnozootechnie, 21, pp. 71-78, Paris.

DONARD E. (1982) - Recherches sur les Léporinés quaternaires (Pléistocène moyen et supérieur, Holocène), Thèse, Bordeaux, $\mathrm{n}^{\circ} 1764$.

DrIESCH A. von den und BoEsSNeCK J. (1970) - Vorgeschichtliche Kaninchen aus zwei südspanischen Siedlungshügeln, Säugetierkundl. Mitt., 28, pp. 127-151.

PietschmanN W. (1977) - Zur Grösse des Rothirsches (Cervus elaphanus L.) in vor- und frühgeschichtlicher Zeit, Thèse, Münich.

POULAIN-JosiEN Th. (1964) - Les animaux domestiques et sauvages en France du Néolithique au Gallo-romain. Étude d'Ethnozoologie à partir des vestiges osseux, Thèse, Paris.

TEICHERT M. (1975) - Osteometrischen Untersuchungen zur Berechnung der Widerristhöhe bei Schafen, Archaeo-Zoological Studies, North Holland-American Elsevier, pp. 51-69. 


\title{
Annexe 2
}

\section{Analyse paléocarpologique : \\ Baume des Anges. Donzère (Drôme), Age du Bronze final}

\author{
par Philippe Marinval
}

Nous avons reçu de Monsieur J. Vital pour analyse quelques paléo-semences provenant de la fosse F. 1 (foyer de la couche 4a) de la grotte de la Baume des Anges, Donzère (Drôme). Elles datent de l'Âge du Bronze final.

Ces macro-restes végétaux furent récoltés par l'archéologue après un tamisage à sec des sédiments fouillés.

Nous avons identifié :

-1 Hordeum vulgare $\mathrm{L}$. (Orge vêtue) $(4,9 \times 2,4$ $\times 1,7 \mathrm{~mm})$.

- 1 Triticum dicoccum $\mathrm{L}$. (Blé amidonnier) $(5,7$ $\times 3,0 \times 2,8 \mathrm{~mm}$ ).

- 1 Triticum aestivum/durum (Blé tendre) $(4,5 \times$ $2,6 \times 3,2 \mathrm{~mm})$.

- 2 fragments de caryopses de Blé attribués au Blé tendre (Triticum cf. aestivum/durum).

Les paléo-semences sont assez bien conservées.

Les trois grains entiers sont représentés sur la figure (fig. 1).

Bien qu'elle soit très réduite, cette découverte est fort intéressante. En effet, nous manquons de données paléocarpologiques en ce qui concerne l'Âge du Bronze final dans la région Rhône-Alpes. Jusqu'à présent, à notre connaissance, il n'existait que l'analyse de Monsieur Coquillat sur les paléo-semences de la Balme-Gontran, Chaley (Ain) (1955).

Les paléo-semences de la Baume des Anges s'intègrent, en tout cas, parfaitement aux données dont nous disposons sur l'alimentation végétale et l'agriculture à l'Âge du Bronze final en France (Marinval, 1983).

Laboratoire de Palynologie, Musée de l'Homme, 75116 Paris
Coquillat M. (1955) - Sur les graines des foyers protohistoriques de la Balme-Gontran, Chaley (Ain). Bull. des Natur. d'Oyonnax, pp. 47-58.

MARINVAl P. (1983) - Approche de l'alimentation végétale du Néolithique au Second Âge du Fer d'après les macro-restes végétaux. Mémoire du diplôme de l'E.H.E.S.S., 284 p., 52 fig., 8 tabl., Paris.
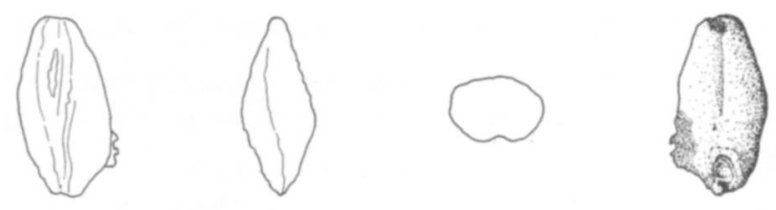

A
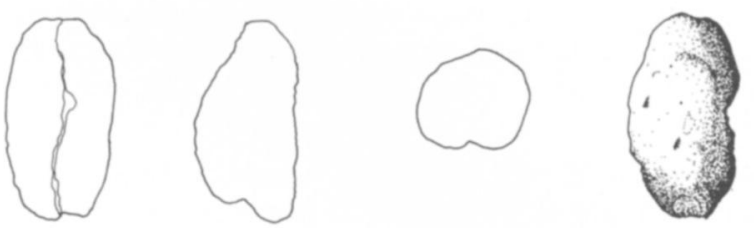

B
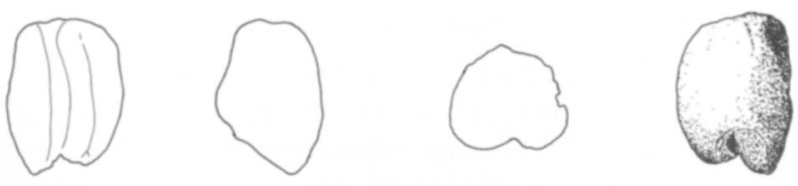

C

A. Hordeum vulgare.

B. Triticum dicoccum.

C. Triticum aestivum/durum. 


\title{
Annexe 3
}

\section{Analyse des charbons de bois de la grotte de la Baume des Anges}

\author{
par Stéphanie Thiebault
}

L'analyse des charbons de bois issus du gisement de la Baume des Anges s'est effectuée sur cassure fraîche au microscope optique à réflexion; elle a permis l'identification de 275 charbons répartis sur trois couches archéologiques distinctes :

C $4 \mathrm{a}$ - attribuée au Bronze final $3 \mathrm{~b}$ (nous avons réuni les 2 prélèvements de cette couche, à savoir : $\mathrm{C} 4 \mathrm{a}$ tamisage qui a livré 6 espèces différentes sur 103 échantillons et $\mathrm{C} 4 \mathrm{a}$ 《 ramassage à la fouille » qui a permis l'identification de 15 espèces réparties sur 79 échantillons.

C 4c - niveau attribué au Bronze final, 55 échantillons, 1 espèce.

C 5 - couche du Bronze final 2, 38 échantillons, 8 espèces.

Les résultats de ces identifications sont regroupés dans les tableaux I et II.

Le tableau I : rassemble toutes les essences reconnues et leur répartition dans les trois niveaux étudiés.

\begin{tabular}{|c|c|c|c|c|c|c|}
\hline \multirow{2}{*}{ Essences } & \multicolumn{2}{|c|}{$c$ a } & \multicolumn{2}{|c|}{$\therefore 4 c$} & \multicolumn{2}{|c|}{$c 5$} \\
\hline & $N$ & 3 & $=$ & $=$ & 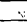 & ב \\
\hline Pinus silvestris & 1 & 0,5 & & & 17 & 44.7 \\
\hline Tarus baccata & 1 & 0.5 & & & & \\
\hline Acer compestre & 9 & 4,9 & & & 2 & 5,2 \\
\hline Acer opulifolium & 4 & 2,1 & & & & \\
\hline Busus sempervirens & 58 & 31,8 & 55 & 100 & 4 & 10,5 \\
\hline Corylus avellana & 2 & 1 & & & 1 & 2,6 \\
\hline Crataegus monogyna & 5 & 2.7 & & & & \\
\hline Evonymus europaeus & 2 & 1 & & & & \\
\hline Fraxinus excelsior & 3 & 1.6 & & & & \\
\hline Phillyrea latifolia & 11 & 6 & & & 1 & 2,6 \\
\hline Populus $s p$. & 9 & 4,9 & & & & \\
\hline Quercus ilex & 33 & 18,1 & & & 10 & 25 \\
\hline Quercus pubescens & 8 & 4,3 & & & 3 & 7,8 \\
\hline Quercus robur-petraea & 31 & 17 & & & & \\
\hline Ulmus campestris & 3 & 1,6 & & & & \\
\hline Viburnem $s p$. & 2 & 1 & & & & \\
\hline TOTAL: & 182 & & 55 & & 38 & \\
\hline
\end{tabular}

Tableau I - Dénombrement et pourcentage des bois carbonisés issus du gisement de la Baume des Anges (Drôme).
Tableau II : diagramme anthracologique des principaux taxons, il tente de mettre en valeur l'évolution de quatre essences jugées importantes dans l'histoire végétale régionale. Sous le terme de Quercus f.c, Chênes à feuillage caduc, nous avons réuni les Chênes pubescents (Quercus pubescens) et les Chênes type roburpetraea.

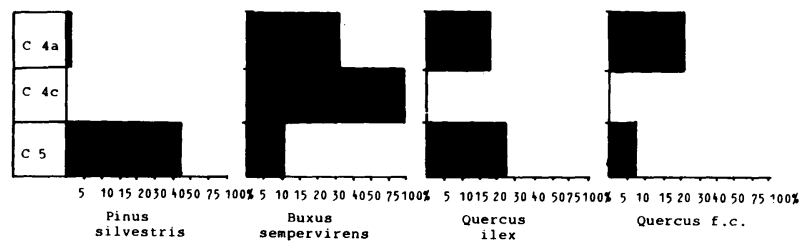

Tableau II - La Baume des Anges (Drôme). Diagramme anthracologique des principales essences.

L'étude anthracologique des sites du Défilé de Donzère offre de multiples intérêts : tout d'abord leur position géographique ; situés sur la rive gauche du Rhône, à un peu plus de $100 \mathrm{~km}$ à vol d'oiseau de la Méditerranée, ils sont encore soumis à une influence méditerranéenne ; leur étude paléobotanique peut donc prendre place parmi celles entreprises dans le Nord de la Provence et les Préalpes Sudoccidentales (Thiebault, 1983).

Outre l'emplacement du gisement, les résultats de l'analyse anthracologique sont à noter : en effet, il est assez rare d'avoir 15 espèces différentes dans une couche attribuée au Bronze final, dans un paysage qui a déjà fortement subi l'influence humaine. Le nombre élevé des espèces en $C 5(8)$ et $C 4 a(15)$ apporte une somme d'informations sur le paysage forestier et le climat local ; en effet, la diversité des essences montre que nous sommes en présence d'un échantillonnage des bois par l'homme, autour du site, et non d'un ramassage dirigé, comme pour le Buis par exemple, dans la couche C $4 c$. 
Après une rapide analyse, nous pouvons dire que le paysage de la Baume des Anges au Bronze final était constitué d'une Chênaie mixte à Chênes à feuillage caduc, Chênes verts, Érables et Noisetiers. Les influences méditerranéennes se traduisent par la présence du Chêne vert, du Buis, du Filaria, essences thermophiles, mais l'If et le Pin sylvestre apportent une note montagnarde et humide.

Soulignons l'importance du Pin sylvestre qui atteint $45 \%$ en $\mathrm{C} 5$ (les Chênes à feuillage caduc forment $32 \%$ des essences) ; son pourcentage décroît pour n'être plus que de $0,5 \%$ en C $4 \mathrm{a}$, remplacé par les Chênes à feuillage caduc : $21 \%$, Chênes verts : $18 \%$ et Buis : $31,8 \%$.

Au Bronze final 2 et $3 b$, Buis et Chêne vert traduisent la dégradation des terres sans doute due à l'homme. Ces essences ont été reconnues sur tout le pourtour méditerranéen occidental comme des essences de dégradation ou de reconquête des terres exploitées par l'agriculture et l'élevage. Le schéma déjà constaté sur de nombreux sites de cette vaste région [(à savoir 4 phases de l'évolution de la végétation au Postglaciaire en corrélation avec les cultures humaines) Vernet, Thiebault et Heinz, 1984]. Le plus proche étant celui d'Unang (Vaucluse) se retrouve plus au nord. Il s'agit ici de la phase 4 qui caractérise le maximum de la détérioration des sols (C 4a : Buxus : $31,8 \%$ et Quercus ilex : 18,1\%).
Signalons enfin l'absence totale du Hêtre. C'est pourtant à partir du Subboréal qu'il quitte ses refuges et se répand, à la faveur d'un climat humide, dans les stations du sud et sud-ouest.

En définitive, au terme de ce rapide survol des résultats anthracologiques nous insisterons sur le fait suivant : cette étude offre un grand intérêt car elle s'inscrit dans l'ensemble des travaux concernant le gradient paléofloristique sud-nord. De fines comparaisons devront être effectuées avec des sites régionaux proches, Unang par exemple.

La diversité et la multiplicité des essences reconnues contribuent à une meilleure connaissance du paysage forestier tout comme des rapports de l'homme et du milieu végétal.

Stéphanie THIEBAULT,

19 , rue Juge, 75015 Paris

Thiebault S. (1983) - L'homme et le milieu végétal à la fin du Tardiglaciaire et au Postglaciaire : analyses anthracologiques de six gisements des Préalpes Sud-occidentales, Thèse $\mathrm{III}^{\mathrm{e}}$ cycle, Paris I, 215 p., 74 fig., 12 tabl., 5 pl.

Vernet J.-L., Thiebault S. et Heinz C. (1984) - Nouvelles données sur la végétation préhistorique postglaciaire méditerranéenne d'après l'analyse anthracologique, Actes du colloque "premières communautés paysannes en Méd. occidentale", CNRS, Montpellier, 1983 (à paraître).

\section{CONSEIL D'ADMINISTRATION DE LA SOCIÉTÉ PRÉHISTORIQUE FRANÇAISE POUR L’ANNÉE 1986}

\section{I. - Présidents d'Honneur}

Mlle H. Alimen. Directeur de recherche au C.N.R.S. ; MM. A. LEROI-GourHaN, professeur au Collège de France ; R. JofrRoy, conservateur en chef honoraire du Musée des Antiquités Nationales ; P.-R. GIOT, Directeur de recherche au C.N.R.S. ; H. DELPORTE, conservateur en chef du Musée des Antiquités Nationales ; G. BAILloud, Maître de recherche au C.N.R.S., ancien secrétaire général de la S.P.F.

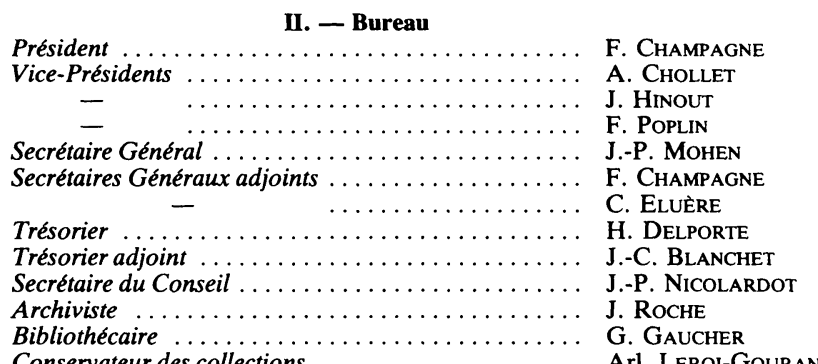

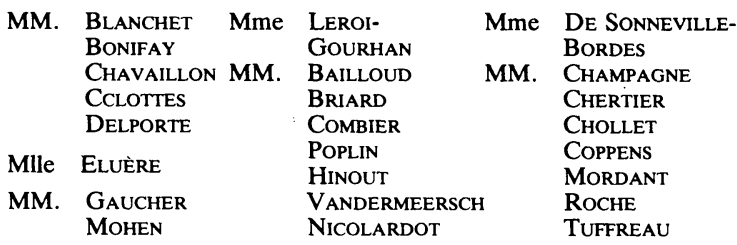

Conseil juridique : $\mathrm{M}^{\mathrm{e}}$ Pierre Maurice-Garçon Notaire : $\mathrm{M}^{e}$ BLANCHET, rue de Beaujolais, Paris

1. Tiers renouvelable en décembre 1986

2. Tiers renouvelable en décembre 1987

3. Tiers renouvelable en décembre 1988

IV. - Membres d'Honneur : MM. P Biberson ; Dr Jullien.

Anciens Présidents de la S.P.F. : E. Rivière, L. Bonnemère, A. De Mortillet, Dr Ballet, Dr Baudoin, A. Guebhard, Dr Henri-Martin, L. Coutil, A. Vire, Dr Atgier (1914-1918), J.-A. Le Bel, A. Vire, Pages-Allary, P. de Givenchy. J.-A. Le Bel, Dr R. De St-Périer, G. Courty, Dessailly, L. Coutil, Dr F. Regnault, G. Poisson, A. Vayson de Pradenne, P. Royer, Colonel Vésigne, Dr P. Rivet, E. Vignard, St-Just Pequart, Abbé H. Breuil, A. Cabrol, Colonel Pupil, H. Desmaisons, Dr Stephen-Chauvet (1940-45), L. Courtier, M. Exsteens, Colonel Vésignié, Mile H. Alimen, J. Blanchard, G. Gaudron, A. Leroi-Gourhan, L.-R. Nougier, Abbé H. Breuil, Dr A. Cheynier, C. Arambourg, Dr R. Jullien, M. Chassaing, R. Joffroy, mile H. Alimen, P.-R. Giot, Mlle G. Henri-Martin, J.-J. Hatt, L. Balout, R. Joffroy, J. Chavaillon, R. Grosjean, H. Delporte, G. Bailloud, Mme A. Leroi-Gourhan, J. Briard, J. Hinout, F. Champagne, Mme de Sonneville-Bordes, Y. Coppens, A. Chollet, J. Roche, E. Bonifay, J. Clottes, J.-P. Mohen, B. Vandermeersch, G. Gaucher. 